\title{
A parametric approach to logistic control within manufacturing simulation
}

\author{
Journal International Journal of Industrial and Systems Engineering \\ Publisher Inderscience Enterprises Ltd \\ ISSN $\quad 1748-5037$ (Print) \\ Issue $\quad$ Volume 5, Number 3/2010 \\ Pages 313-326 \\ DOI 10.1504/IJISE.2010.031963
}

Online Date Thursday, March 04, 2010 


\title{
A PARAMETRIC APPROACH TO LOGISTIC CONTROL WITHIN MANUFACTURING SIMULATION
}

Richard Farr $^{1}{ }^{*}$, James Tannock ${ }^{1}$, Torgny Almgren ${ }^{2}$

'Operations Management Division, University of Nottingham, Nottingham NG8 1BB, UK

Fax: +44 115 8467855, E-mail: richard.farr / james.d.tannock@nottingham.ac.uk

2 Operations Planning and Logistics, Volvo Aero, 46181 Trollhättan, Sweden

Fax: +46 520 98590, E-mail: torgny.almgren@volvo.com

\begin{abstract}
Simulation offers a powerful way to investigate the boundaries of what can be achieved by a manufacturing system, demonstrating the impact of schedules and policy decisions such as the placement of buffers and the size of safety stocks. Unfortunately, the construction of factory models at the necessary level of detail is a time-consuming process, requiring specialist skills.
\end{abstract}

This paper describes a novel approach to the representation of logistic control in the simulation of a business unit at Volvo Aero in Trollhättan, Sweden. Several innovative features were built into the model, making it possible for users who were not simulation experts to explore a broad range of scenarios. The resulting model architecture, as described in this paper, takes simulation out of the computer lab and places it in the hands of managers, as an enabler of the responsive enterprise.

Keywords: Parametric simulation, logistic control, kanban, aerospace manufacturing

\section{Biographical Notes:}

Richard Farr is a senior research fellow in the Operations Management Division at the University of Nottingham. He obtained a B.Eng in Manufacturing Management, and his PhD, from the Aeronautical and Mechanical Engineering Department at the University of Salford. His research interests include simulation interfacing, mechatronics manufacturing, and strategic capacity acquisition and retention.

James Tannock is Reader in Quality and Operations Management at the University of Nottingham. His research Interests include quality management and the supply chain in developing and newly industrialised countries, simulation techniques for manufacturing quality and the supply chain, quality data management, and artificial intelligence methods for process monitoring, control and improvement.

Torgny Almgren is company specialist in R\&D Logistics within the Operations Planning and Logistics Department at the Volvo Aero in Trollhättan, Sweden. His research interests include the modelling of logistic systems and the application of optimisation methodologies to logistics (primarily production planning and inventory management). His $\mathrm{PhD}$, addressing production planning in a mining environment, was obtained from Luleå Technical University in 1994.

\footnotetext{
* Corresponding Author
} 


\section{Introduction}

Located in Trollhättan, Sweden, Volvo Aero Corporation develop and manufacture a number of engine components, supplying Rolls-Royce, General Electric, Pratt \& Whitney and other aerospace primes. They typically produce large components of considerable complexity, existing in the middle of a supply chain. The specialised nature of this work means that volumes are relatively low, while the value of each component is substantial. Thus, it was desired to explore the potential of alternative methods of logistic control, to reduce tied-up capital in the form of inventory, without impacting upon delivery performance.

A case study facility was selected, producing two kinds of Turbine Exhaust Casing (TEC). These are large structures with features that must be produced by casting or fabrication, plus a considerable amount of machining. The facility is operated as a focused factory; originally advocated by Skinner (1974), a focused factory concentrates upon a limited set of products in order to avoid the contradictions and compromises introduced when trying to bid for every business opportunity. The two TECs that are made here are for the International Aero Engines V2500 (as used in aircraft such as the Airbus A320 family) and the Pratt \& Whitney 2000 (Boeing 757 and C17 Globemaster). Production volumes for the two components differ although the methods are broadly similar.

The market for aero engines is unusual in a number of ways, combining high value products, low volume, a long product lifecycle and a lengthy support requirement in the aftermarket. It is also a market that has exhibited sudden demand changes in response to oil price increases, health scares, wars and terrorism (ACARE, 2004), while further complications are introduced by the behaviour of airlines, including usage patterns, the 
desire to standardize equipment within fleets, and the trade in used aircraft and engines. The net result is that demand patterns are variable and difficult to predict.

It was not the aim of this research to 'optimize' the manufacturing system in any simple sense. It was felt that any attempt to produce an ideal solution would simply produce a fragile one that could not be exposed to the reality of the aerospace value chain. As Seppala and Holmstrom (1995) observed, a supply chain made up from a set of optimized subsystems will not perform optimally together. Further, as Ingalls (1998) pointed out, variability makes optimization impractical, whether arising from demand forecast variance, supply reliability, or the quality of incoming material. Davis (1993) classified the sources of uncertainty similarly, citing problems of supplier performance, manufacturing system performance, and demand irregularity. The effects of all such issues could potentially be explored using the simulation that was developed, but the goal was not to find optimal parameters. Located in the midst of a complex supply chain, the case study facility was largely at the mercy of external events. Thus, any attempt to produce a better system would require a tradeoff between a number of goals, including leanness, robustness and delivery performance.

Although the requirement for TECs can fluctuate, the period when the study was conducted was one of strong growth in demand, and in the first instance it was desired to explore the possibility of increasing the throughput of the facility. Much of the production equipment was very expensive, so any solution that required machines to be duplicated would have introduced a cost burden to be borne during periods of low demand. However, bottleneck machines were already operating on multiple shifts, so any improvement in the throughput or on-time delivery performance of the focused factory would have to come about through improved logistic control. 
The overall effect of the various practical issues described above is to complicate the process of simulation model construction and validation, by potentially requiring the construction of many models incorporating slightly different logistic control mechanisms to explore different scenarios. The requirement for a combination of a high degree of practical and theoretical understanding of the manufacturing facility, with a knowledge of simulation theory and methods, had previously meant that little useful progress had been made. In this area the literature is of little help. Hence, the authors adopted a parametric approach to the simulation of the focused factory.

\section{Potential Benefits of Simulation}

Simulation allows the performance of a system to be investigated, using a model so that the real-world facility is not disturbed. If a suitable replica of a business unit can be created, many situations can be investigated, and planners can experiment with system parameters to reduce tied-up capital, increase throughput or increase confidence in the assurances they give to customers. Robinson (2004) shows how discrete event simulation can provide significant insights into how a system will perform and how it might be improved. This demands a systematic approach that begins with a suitable conceptual model, implementation with appropriate software tools, validation, and experimentation that takes the model's inherent limitations and assumptions into account. Careful management of users' expectations is also necessary (Robinson and Pidd, 1998).

Development of simulation models to this level of quality requires substantial effort. An analyst may spend several months constructing and validating a detailed model of a manufacturing system or a supply chain. While such a model can be useful, the lead-time and cost inherent in its creation limit its utility. Tools and techniques that can reduce the time that must be spent in model development are thus of considerable interest. One 
approach with considerable potential to reduce model development time is that of datadriven model construction, where a software tool creates a model directly from company ERP data (Tannock et al, 2007). This has been seen to create valid models of supply chains that can subsequently be used to examine their performance.

One of the advantages attributed to simulation is that it is possible to gain understanding of a system that does not (yet) exist in reality (Carson, 2005). The capability to examine the performance of future systems is of interest not only in the study of conceptual supply chains such as the work described in (Tannock et al, 2007), but also in less radical conceptual systems. For instance, manufacturers are likely to find it rewarding to study the performance of a present-day facility when operating under an alternative system of logistic control.

Logistic control encompasses all the decision-making stages and systems that can influence the flow of products through a manufacturing system, from the macro-level where assets are selected and arranged, through decisions affecting process plans and operating procedures, down to the micro-level where formal or informal rules are employed in the day-to-day operation of the plant. Production planning is a component within this, as are buffers and systems that control the flow of products. All such elements must be viewed as components within a complex system.

Where an MRP system is currently used for production planning and control, it might be desired to see how the factory might perform if controlled by other means, such as Kanban (Ohno, 1988). A change to the logistic control method might reduce stock levels, reduce lead times, and/or improve customer service - although the outcomes are by no means guaranteed. Krajewski et al (1987) used simulation to study the potential of Kanban in traditionally organized businesses in the USA, finding that it was by no means a panacea. 
The application of a computer-based simulation to such a study has clear advantages in that it will not disrupt the operation of the real system, and it allows certain variables to be experimented with, such as the level of safety stocks, the size of buffers or the standard lead times used to issue orders. Simulation also offers the opportunity to repeat an experiment many times - a technique not normally available to the management scientist (Pidd, 1988). Thus, the approach has considerable potential here, but a problem remained, in the cost and time required to develop and validate suitable models. Effectively, under conventional manual approaches to simulation, each system of logistic control that is to be investigated demands the creation of a separate model - with consequences for its validation, and use thereafter.

In order to overcome this limitation, a parametric approach to logistic control was developed. This method and its advantages are demonstrated using experimentation with a validated simulation from the case study company.

\section{THE SIMULATION APPROACH}

Arena from Rockwell Software was selected for the construction of the simulation. Whereas many simulation tools attempt to reproduce a layout of the facility under study, Arena uses a flowchart paradigm, where each entity is an event rather than a work centre. This matched the existing 'Operations Flow Diagram' that was used at Volvo Aero to illustrate the process plan for each TEC. It was useful that the layout of the Arena model could be made to resemble this, because a strong correlation between the model and Volvo Aero's documentation helped in obtaining 'buy-in', and eliciting comments from staff. Figure 1 shows the resulting model layout.

Historical data had been gathered from the beginning of 2004 onwards, recording the time and date when each workpiece had left each production stage. The launch dates for raw materials and the completion dates for finished goods were also known. Analysis of 
these historical data revealed that the duration of most operations could be expressed as a triangular distribution, representing natural variability in processing times and taking into account the incidence of minor problems. Major problems that caused a workpiece to be taken out of the manufacturing sequence formed a 'tail' on some distributions; these were handled separately so that the impact of quality problems could be investigated in closer detail.

Although the modelling task was substantial, most of the activities that were represented fell into just a few categories, such as machining, transportation and joining (assembly or welding) operations. In each case, the activity could be represented via a 'submodel'. These are self-contained collections of Arena entities which, once developed and tested, could be used repeatedly, given unique names and appropriate parameters including the resources required and the processing time. Thus, although the eventual model consists of almost 1500 interlinked elements, most were within proven submodels.

The earliest modelling activity focused upon achieving an 'as-is' representation of the focused factory's existing logistics control system. This was an MRP-driven system, using due dates and a standard lead time to determine when materials should be launched into the facility. Originally, the launch dates for each component were held within an array, although it was found that the functions of the MRP system were more easily represented within an Excel spreadsheet. From this, the quantity of parts to be launched on each day were read into the simulation model automatically, each time it ran. This had the advantage that the user was no longer required to be an Arena expert in order to create experimental scenarios, increasing the usefulness of the model. A further refinement provided a means for defining the work-in-progress (WIP) at each workcentre at the start of a model run. The focused factory did not start out empty when data collection began in 2004; production of both TECs was already well underway. Simulations can generally be started empty, and if 
left to run long enough are likely to reach a steady state (if the system has a steady state). However, by taking advantage of the historical data provided by Volvo Aero, the model was populated with workpieces at various stages of completeness at 'time zero'. As a result, it was found that the model would reach a realistic steady state much more quickly, this being observed at around seventy days where an empty model only achieved a steady state after almost two hundred days. When performing a large number of experiments with a complex model, this led to considerable time savings.

Validation is an essential pre-requisite to the use of any simulation in experimentation to understand and improve the performance of the system (Robinson, 2004). The 'MRPdriven' model provided a useful means of validation, since the performance of the simulation could be compared with the production history of the real system. This process also served to fine-tune the model and tackle some issues of accuracy. Naturally, some differences were observed; at first the simulation was found to perform far worse than the real system. At peak demand, lead times would increase steadily, as would the amount of WIP in the system as it became choked with material. Investigation eventually revealed that some of the information that had been supplied, describing the operating practices for key machine tools, was out of date since a means had been found to increase their effective capacity. When the model was changed to reflect the new practices, the results resembled those of the real system. Performance metrics that were used for model validation included lead time, fill rate, level of WIP and the utilization of selected machines. All could be compared with historical data, and with results from static simulation, deriving capacity mathematically, given typical processing times. This technique, plus experience within the facility, suggested conditions then prevailing would result in a maximum output of twelve TECs per week, and this was borne out via experimentation. 


\section{THE METHOD OF PARAMETRIC LOGISTIC CONTROL}

It should be possible to simulate any logistic control system within a suitably-designed model, and it was a goal of this work to examine several such systems. As described above a spreadsheet had already been employed with benefit, to provide inputs to the original, MRP-driven model. This approach was then extended, such that named ranges within the spreadsheet held information to be used during each model run, defining the initial placement of inventory at the start of the model run, schedules for the arrival of raw materials, and so on. This was an elegant and accessible way to construct scenarios because it did not require user knowledge of any tool more complex than Microsoft Excel, for alternative scenarios to be defined.

By further developing this approach, a means was developed whereby the logistic control strategy could also be described parametrically, allowing experiments to be conducted under 'push', 'pull' and hybrid control systems. Under the original representation of the MRP system used by Volvo Aero, the only parameter that could be adjusted to influence the performance of this system was the standard lead time; the offset between when a product is due and when work should commence (or when an order should be dispatched upstream). This calculation was performed within the spreadsheet; and hence the MRP-driven model simulated a planning, not a control system, so that when a disruption occurred the system did not react well. For example, when a bottleneck developed, the component launch schedule was not changed, and the result was lead times and WIP levels that tended to increase rapidly.

Small test simulation models previously created by the authors within Arena had demonstrated how several systems of logistic control might be represented, the following being created: 
- An MRP schedule-based 'push' system - analogous to the existing system in the case-study factory

- A Kanban system (Ohno, 1988) modelling the flow of tokens that had to be present at a workcentre before an operation would commence

- A Kanban squares system, where an operation was triggered when a space developed in a buffer immediately downstream

- A hybrid drum-buffer-rope system (Goldratt, 1980; Goldratt and Cox, 1987) with elements of both 'push' and 'pull' logistic control, centred upon the most constrained resource.

It was desired to apply these alternative systems of logistic control to the validated model. Under a conventional approach to simulation, this would have meant copying the existing, MRP-driven model, and adapting it to include new workflow control logic, encapsulating the rules of a particular system of logistic control. Within Arena, this involves the addition of signalling modules, and resources or buffers that wait for such signals.

Of course, making any such change moves the developer away from the originally validated model, reducing confidence in the validity of the modified model. Each such development also requires additional time, and could introduce errors.

In a novel approach, a form of logistic control for simulations was developed where changing model parameters allowed the system to exhibit the behaviour of a 'push' system, a 'pull' system or a hybrid. In this way, the merits of each approach could be evaluated. To achieve this, 'pull' style logistic control logic was added to the existing MRP-driven simulation. It made use of the triggering method developed for the 'Kanban squares' system, but instead of placing Kanban squares after every machine (the high cost of populating the buffers with WIP could not be justified), they were located at the end of a 
sequence of operations. Figure 2 shows a simplified example of this. The sequences were chosen to match those used at Volvo Aero, on whiteboards that showed the status of the facility. These sequences resembled Period Batch Control (Burbidge, 1988; Benders and Riezebos, 2002) in that a group of processes of different duration were combined into groups with similar overall duration. With the groupings used, a workpiece could be expected to advance from one sequence to the next, each week - and therefore we expected each sequence to exhibit a WIP level that was approximately equal to a week's output for each TEC type. (There was one exception; one of the sequences contained several lengthy operations, and was of double duration. This is a permissible feature of Period Batch Control.)

The Kanban squares located at the end of each sequence were not merely a buffer, but also the instrument of logistic control. They only released components into the sequence downstream if the level of WIP within that sequence (including its own buffer) had fallen below a target figure. This was related to the ConWIP system of logistic control (Spearman et al., 1990), in that it limited the total number of workpieces that are permitted to be in a system, although in this case the level of WIP permitted was for a short sequence of operations rather than for the system as a whole. Also, it should be noted that the sequence described a set of operations which need not relate to a physical grouping of machines; nor does the method of logistic control need to be the same for different product types.

The parameters specifying a target level of WIP for each sequence determined their leanness, being in effect the number of kanbans circulating within that area of the factory. In reality, there was no need to model the movement of tokens within the simulation; keeping track of the number of workpieces within a sequence and comparing this with the target figure achieved the same result. The target levels for WIP were specified within the same spreadsheet that detailed the delivery schedule, standard lead times, raw material 
availability, etc. Thus, we had produced a factory simulation system that could be configured by anybody who could use a spreadsheet, rather than one that required the involvement of an analyst. In such a system it became very simple to change between representing a 'push' or 'pull' system of logistic control. For example, if the user specifies a target for WIP that is very high (perhaps 9,999) then the preceding buffer will always allow parts through. In effect, that sequence becomes a 'push' system. Conversely, by specifying a low target WIP figure, the user imposes control and can then explore the consequences in terms of lead times, fill rates and tied-up capital, etc. The method also allows the modelling of hybrid systems, where some sequences are 'pushed' (controlled solely by the arrival of raw materials) and others are 'pulled'. As such, if the bottleneck process can be identified, it is possible to model a form of Optimized Production Technology (OPT), as described by Goldratt (1980).

Fifteen different scenarios were explored in the first programme of experiments; three different systems of logistic control ('Push', 'Pull' and OPT) were each subjected to five different 'problems' (steady state, fluctuating demand, component shortage, machine breakdown and quality problem). These could then be compared to see which system of logistic control provided the best mix of leanness, robustness and responsiveness.

\section{RESULTS AND DISCUSSION}

The Arena model was run, with appropriate entries in the spreadsheet to represent the fifteen different scenarios, and the results were collated. The results presented here relate to the 'component shortage' problem scenario, and show how the business unit could be expected to cope with a problem of this kind, under three different systems of logistic control. Each suffered exactly the same shortage. In Figure 3, the cumulative fill rates can be seen, showing that the 'pull' scheduled system responded best, in terms of maintaining the fill rate for longer, and then returning to the delivery schedule more quickly. It can 
therefore be said to be more robust and resilient. Under a steady state, where there were no disruptions to the focused factory, the 'push' system outperformed the 'pull' one, but by holding components within sequences or buffers, the 'pull' version demonstrated real value in an uncertain environment.

In Figure 4, the level of WIP held within the facility is shown. Here, a problem with the 'push' system can be seen, as it failed to react to the non-arrival of outer rings. Weld assemblies continue to be amassed, despite the fact that they could not be completed until the supply of outer rings was restored. The 'pull' and OPT systems did not exhibit this flaw.

The poor fill rate and slow recovery shown by the OPT system during this experiment should not be taken to mean that this system of logistic control is undesirable. In effect, under parametric logistic control, there are not merely three different systems of logistic control to examine, but hundreds, since the experiment could be repeated with different target levels for WIP in one or more of the sequences. In this way, further tradeoffs between leanness and robustness would be explored. This ability to fine-tune the manufacturing system is useful, but it means that generic comparison of 'push' and 'pull' methods of logistic control are of limited value.

Indeed, the circumstances of the focused factory meant that a 'textbook' implementation of some system of logistic control was impractical. Given the low volume of parts processed, workflow tended to be 'lumpy' (which is to say, not well suited to Kanban). OPT system implementation was made more difficult because the bottleneck constraint tended to move, with two different product types competing for the same resources. (This also poses problems for a Kanban system, since it was difficult to select the best number of tokens to circulate, although the aggregation of WIP levels in sequences of operations largely negated the problem.) Finally, the mixture of product types with 
different processing times, and some dependence upon resources outside the boundaries of the focused factory, complicates any notion of controlling operations with a drumbeat.

While 'pure' exemplar facilities do exist, it must be recognised that many businesses already operate a partial or hybrid implementation of a system of logistic control, where some areas of their facility are leaner than others. Economic order quantities and economic batch sizes at certain machines may well influence the evolution of the system. To the lean manufacturing purist, any such issues offer themselves as a target for process redesign; in the aerospace value chain, however, volumes will always be very low, compared to a highvolume automotive or electronics plant. It was thus necessary to have a means of simulating not only the 'classic' logistic control concepts, but also a variety of hybrids.

Our experiments confirm that a certain level of WIP can have considerable value when a problem develops, in that this allows fill rates to be maintained, keeps machines utilized, and speeds recovery. If it is known that disruptions such as the component shortage shown here are likely to occur, then it might well be worthwhile changing the system of logistic control, with the simulation results used to support a cost-benefit analysis. We do not know exactly how much money a major disruption costs the business. There are usually difficultto-quantify costs associated with remedial actions (overtime, managerial input, making special deliveries, etc.) and perhaps penalty clauses when plans break down. However, a simulation like the one described in this paper allows planners to make better-informed decisions about contingencies and the level of risk they are prepared to tolerate.

\section{Concluding Remarks}

This paper has described a novel, parametric system that allows different logistic control approaches to be applied to a validated simulation model. This allows a wide range of experiments to be conducted, representing different types of control strategy, with various parameters, without altering the simulation model itself. It also allows the 
modelling of hybrid systems of logistic control with multiple 'pull' and 'push' scheduled segments in the operations flow, if desired. This system has facilitated experimentation to investigate the impact of a number of different sources of variability, making use of a broad range of performance metrics. Using the example of a component shortage, this paper has illustrated how a system of logistic control that might not appear to be optimal under ideal conditions can come into its own, in the event of a disruption.

When changes to product designs or the production facility are required, the parametric model also simplifies the process of updating the simulation, since the need for multiple models representing various logistic control scenarios has been eliminated. This reduces the cost and time required, and should help to reduce the incidence of modelling errors.

The initial programme of experiments described in this paper must now be expanded upon, conducting longer model runs that present more complex, realistic challenges. In this way, it is hoped that further insights will be obtained. While it is impossible to predict the exact perturbations to which an aerospace supply chain can be subjected, we have developed a cost-effective means of examining a wide range of hypothetical situations, with confidence in the validity of the results obtained.

\section{References}

ACARE (2004) Advisory Council for Aeronautics Research in Europe, Strategic Research Agenda, Volume 2, available from www.acare4europe.org

Benders, J. and Riezebos, J. (2002) "Period batch control: classic, not outdated", Production Planning \& Control Vol. 13, No. 6, pp497-506

Burbidge, J.L. (1988) "Period Batch Control”, Computer-Aided Production Management, IFIP State Of The Art Reports pp. 71-75

Carson, J.S. (2005) "Introduction to Modelling and Simulation", Proceedings of the 37th Winter Simulation Conference, 4-7 December 2005, Orlando, USA, pp. 16-23

Davis, T. (1993), Effective Supply Chain Management, Sloan Management Review, Vol. 34, No. 4 , pp. $35-46$ 
Goldratt, E.M. (1980) “Optimized Production Timetable: A Revolutionary Program for Industry, Proceedings of the 23rd Annual APICS Conference, Falls Church VA, pp. $172-176$

Goldratt, E.M. and Cox, J. (1987) "The Goal: A Process of Ongoing Improvement" (revised edition), North River Press, New York

Ingalls, R.G. (1998) “The Value of Simulation in Modelling Supply Chains", Proceedings of the 30th Winter Simulation Conference, December 13-16, 1998, Washington DC, USA

Krajewski, L.J., King, B.E., Ritzman, L.P. and Wong, D.S. (1987) "Kanban, MRP, and Shaping the Manufacturing Environment” Management Science, Vol. 33, No. 1, pp. $39-57$

Ohno, T. (1988) "Toyota Production System: Beyond Large-Scale Production", Productivity Press, New York

Pidd, M. (1988) “Computer Simulation in Management Science”, 2nd Ed., John Wiley and Sons Ltd, Chichester, UK

Robinson, S. (2004) "Simulation: The Practice of Model Development and Use", Wiley, ISBN: 978-0-470-84772-5

Robinson, S. and Pidd, M. (1998) "Provider and customer expectations of successful simulation projects" Journal of the Operational Research Society, Vol. 49, No. 3, March 1998 , pp. 200-209

Seppala, J. and Holmstrom, J. (1995) "Rough modelling of logistics networks" Integrated Manufacturing Systems, Vol. 6, No. 5, pp. 13-20

Skinner, W. (1974) “The Focused Factory”, Harvard Business Review, Vol. 52, pp113-121 Spearman, M.L., Woodruff, D.L. and Hopp, W.J. (1990) "CONWIP: a pull alternative to Kanban” International Journal of Production Research, Vol. 28, No. 5, pp. 879-894

Tannock, J., Cao, B., Farr, R. and Byrne, M. (2007) "Data-driven simulation of the supplychain - Insights from the aerospace sector" International Journal of Production Economics, Volume 110, Issues 1-2, October 2007, pp. 70-84

\section{Acknowledgements}

This work has been funded by the European Commission as part of the VIVACE Integrated Project (Sixth Framework Programme, contract number AIP3-CT-2003-502917). The 
authors wish to thank staff at Volvo Aero Corporation for their contribution to the work described.

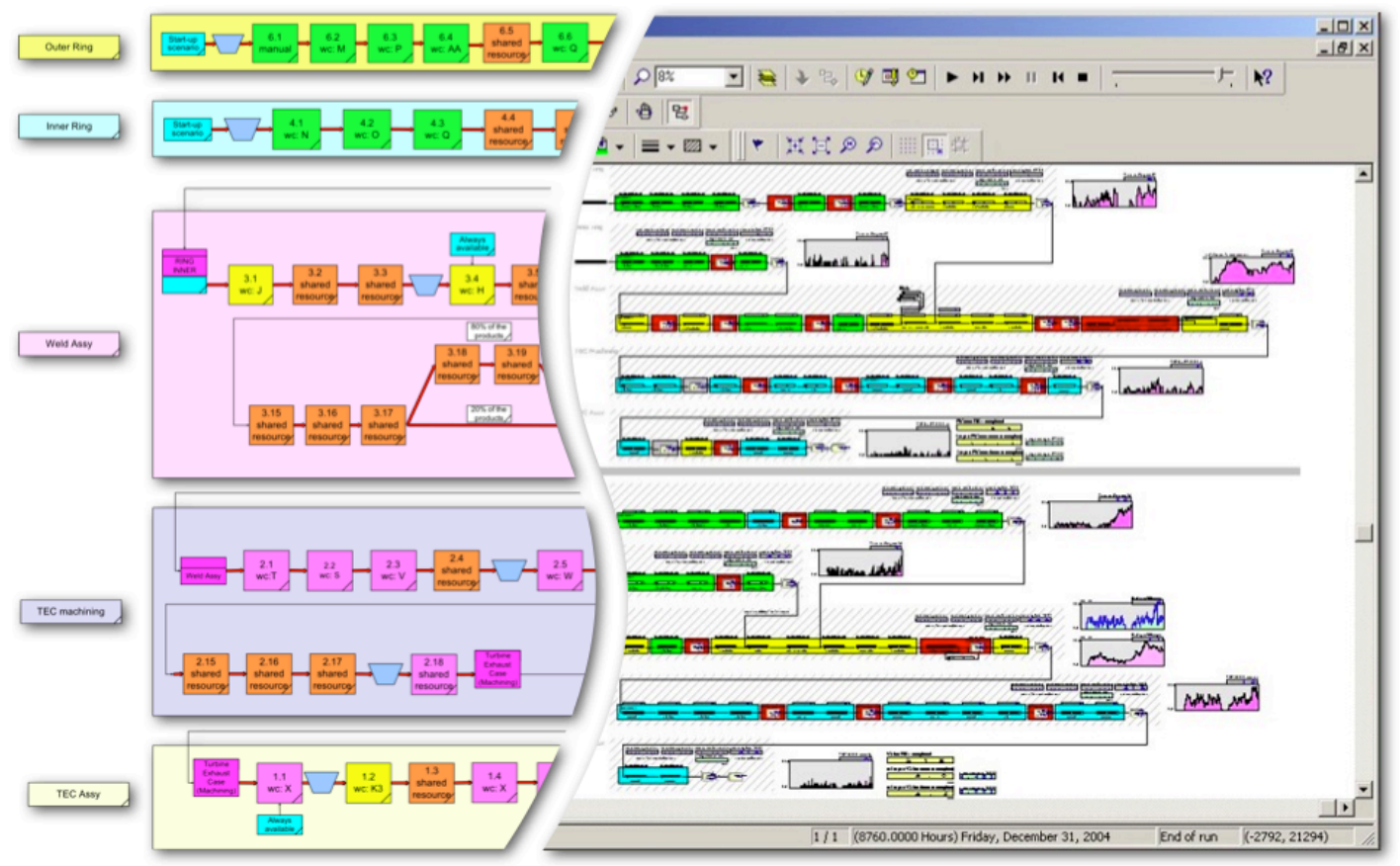

Figure 1: Operations Flow Diagram, and the resulting simulation

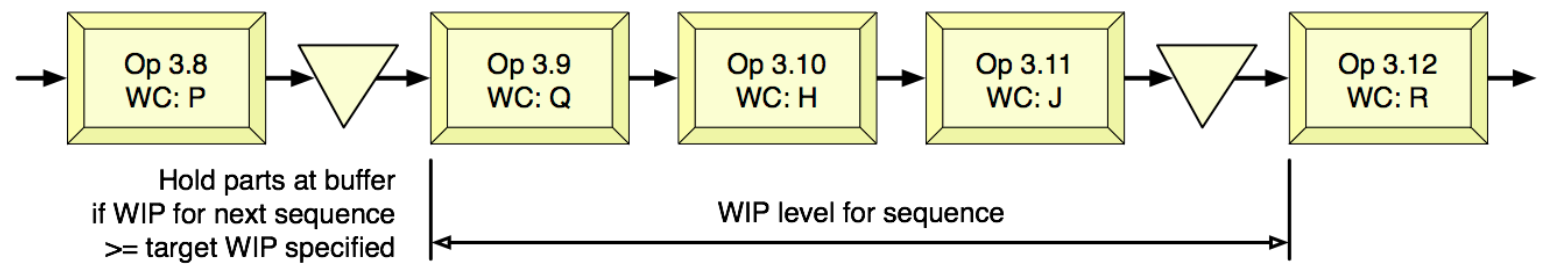

Figure 2: Conceptual model for parametric logistic control 


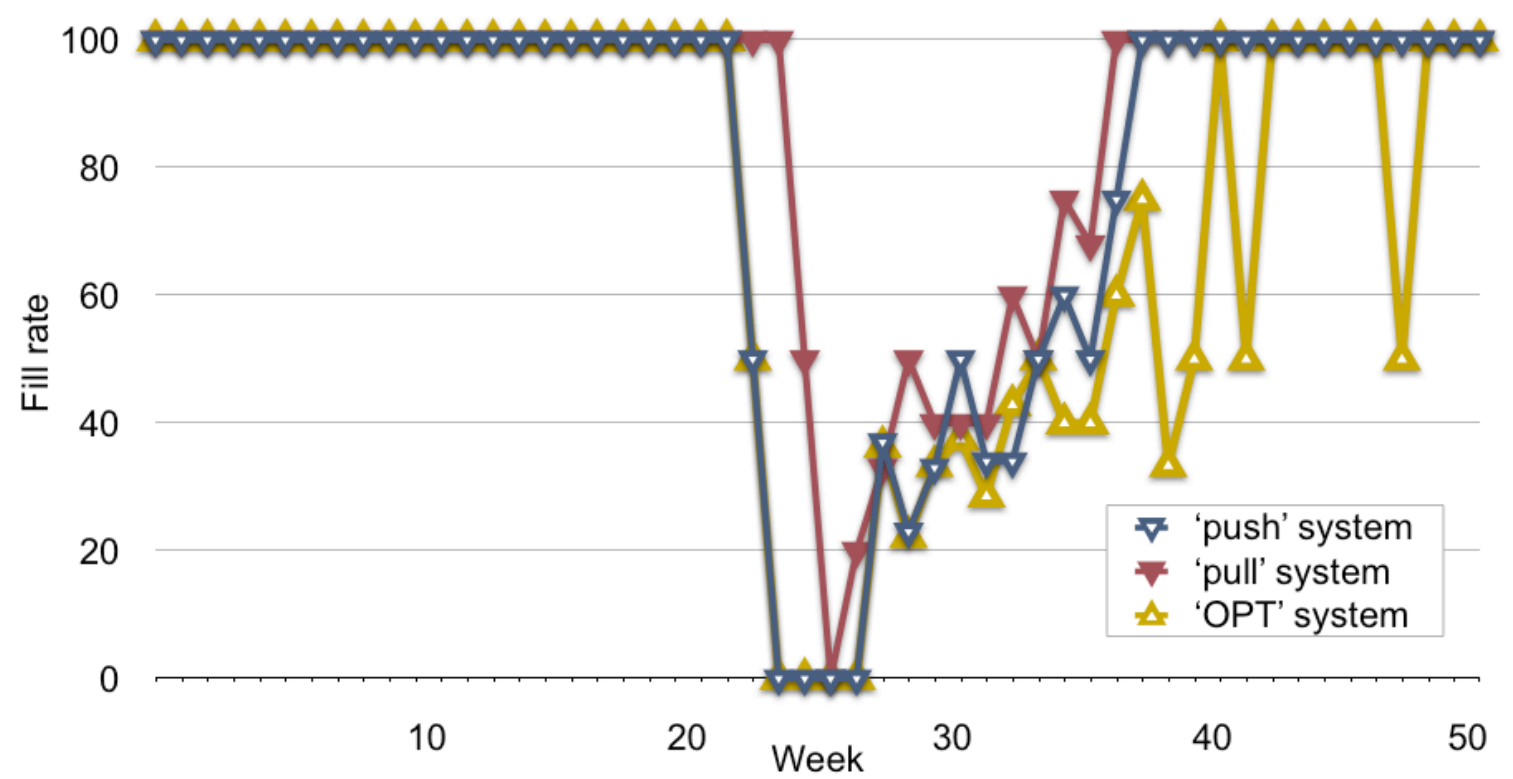

Figure 3: Cumulative fill rate under three systems of logistic control, when suffering an identical component shortage
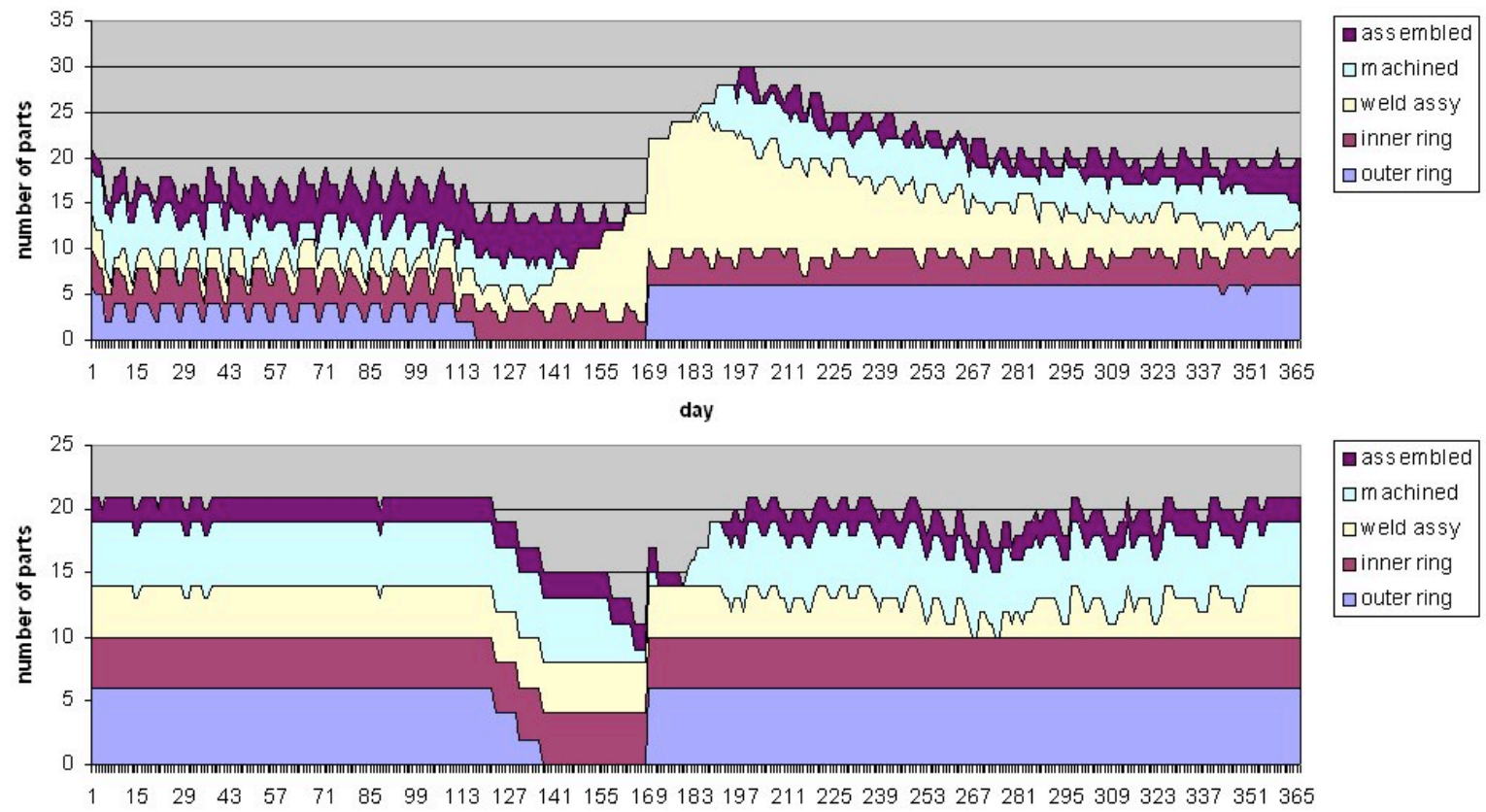

口assembled $\square$ machined 口weld assy 口inner ring outer ring

day

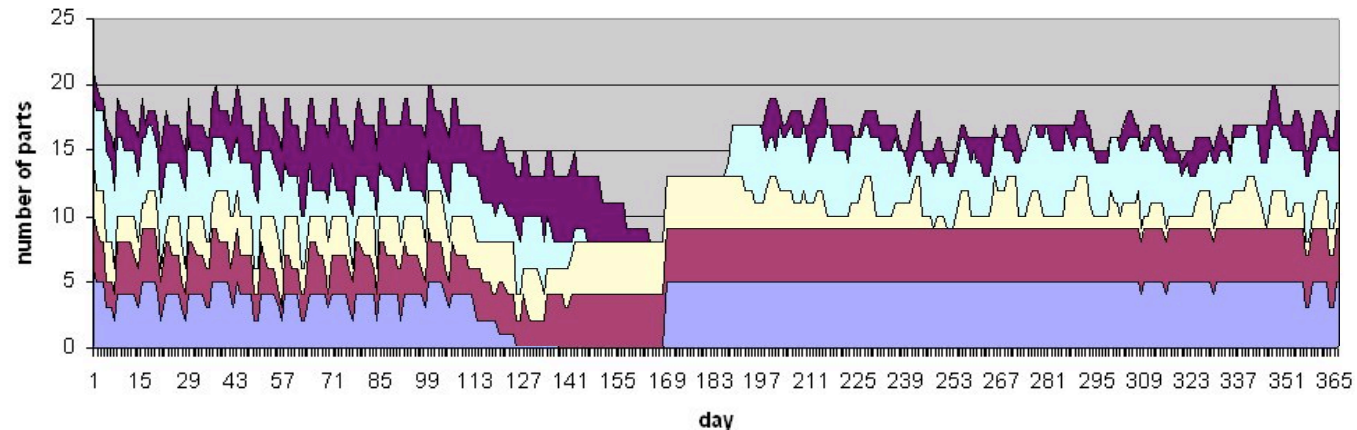

a assembled $\square$ machined 口weld assy øinner ring c outer ring

Figure 4: WIP levels observed under 'Push', 'Pull' and OPT systems of logistic control, when suffering from an identical component shortage 